\title{
Control analysis of the TMT primary segment assembly
}

Peter M. Thompson, Douglas G. MacMynowski, Mark J. Sirota

Peter M. Thompson, Douglas G. MacMynowski, Mark J. Sirota, "Control analysis of the TMT primary segment assembly," Proc. SPIE 7012, Groundbased and Airborne Telescopes II, 70121N (10 July 2008); doi:

10.1117/12.790287

GPIE Event: SPIE Astronomical Telescopes + Instrumentation, 2008, Marseille, France 


\title{
Control Analysis of the TMT Primary Segment Assembly
}

\author{
Peter M. Thompson ${ }^{1 \mathrm{a}}$, Douglas G. MacMynowski ${ }^{\mathrm{b}}$, Mark J. Sirota ${ }^{\mathrm{b}}$ \\ ${ }^{a}$ Systems Technology, Inc., 13766 S. Hawthorne Blvd, Hawthorne, CA USA 90250 \\ ${ }^{\mathrm{b}}$ TMT Observatory Corporation, 2632 E. Washington Blvd., Pasadena, CA 91107
}

\begin{abstract}
The primary mirror control system (M1CS) stabilizes the 492 segments of the Thirty Meter Telescope primary mirror in the presence of disturbances. Each Primary Segment Assembly (PSA) has three actuators and position sensors that control the piston, tip, and tilt of the mirror segment. Requirements for the PSA position controller are presented, with the main requirements being 10 Newton per micron stiffness below one Hertz, where wind is the primary disturbance. Bandwidths of the PSA position controller of about twenty Hertz, assuming a soft actuator, are needed to meet this requirement. A finite element model of the PSA was developed and used for a preliminary control design. PSA structural modes at 40, 90, and 120 impact the control design. We have studied control designs with different actuators, sensors, and structural filters in order to assess disturbance rejection properties and interactions with the PSA structural modes. The performance requirements are achieved using voice coil actuators with modal control architecture for piston, tip, and tilt. Force interactions with the underlying mirror cell are important, and we present the status of our studies of the control structure interaction effect (CSIE). A related paper presents further analysis of the CSIE and MICS global position control loop.
\end{abstract}

Keywords: primary mirror, primary segment assembly, segment support assembly, control structure interaction, control.

\section{INTRODUCTION}

The primary mirror of the Thirty Meter Telescope (TMT) consists of 492 hexagonal segments, whose out-of-plane positions are accurately controlled in the presence of gravity, thermal, and wind disturbances by the primary mirror control system (M1CS). The wind disturbances have the highest frequency content, and thus drive the dynamic requirements for the M1CS. This is in contrast to the primary mirror control system at Keck which only attenuates the low frequency gravitational and temperature disturbances. The in-plane positions of the segments are controlled passively via the Primary Segment Assembly (PSA). Each PSA consists of a mirror segment, axial and lateral support systems, three actuators and 12 edge sensor halves (two per segment edge). The 492 PSA's are rigidly mounted to the top layer of the mirror cell. A diagram is shown in Figure 1a.

The M1CS consists of 492 localized inner control loops with each loop controlling the piston, tip, and tilt of a single segment via three actuators and three actuator position sensors. The overall shape of the primary mirror is controlled via an outer multiple-input-multiple-output (MIMO) global control loop that uses the segment to segment edge sensors for feedback. The edge sensor "set points" for the outer loop are determined off-line using on sky measurements by the Alignment and Phasing System (APS) [Ref. 1]. The mirror cell acts as a backing structure for the multiple PSAs. If the mirror cell was rigid over the frequency range of interest the 492 inner control loops could be considered independent. As a result of the size of the TMT structure along with the requirement to reject wind disturbances the coupling between the segments via the mirror cell cannot be ignored. This coupling effect is known as the control structure interaction effect (CSIE).

This paper focuses on the inner loop servo design of the 492 segments. Idealized voice-coil (soft) actuators are assumed, and the performance is compared to idealized hard actuators. The primary requirement on the inner loop actuator control is to ensure sufficient local stiffness at low frequencies; otherwise the segment response in the presence of wind over M1 would require an unachievable global control bandwidth in order to meet stringent performance targets. The wind disturbance is a result of controlling the thermal environment over the primary mirror; vents will be opened in the enclosure to maintain roughly $1 \mathrm{~m} / \mathrm{s}$ wind speed across M1 introducing turbulence with a characteristic length scale of $\sim 5 \mathrm{~m}$. A companion paper [Ref. 2] describes the global M1CS loop.

${ }^{1}$ pthompson@systemstech.com; phone (310) 679-2281; fax (310) 644-3887; www.systemtech.com

Ground-based and Airborne Telescopes II, edited by Larry M. Stepp, Roberto Gilmozzi, Proc. of SPIE Vol. 7012, 70121N, (2008) -0277-786X/08/\$18 - doi: 10.1117/12.790287 


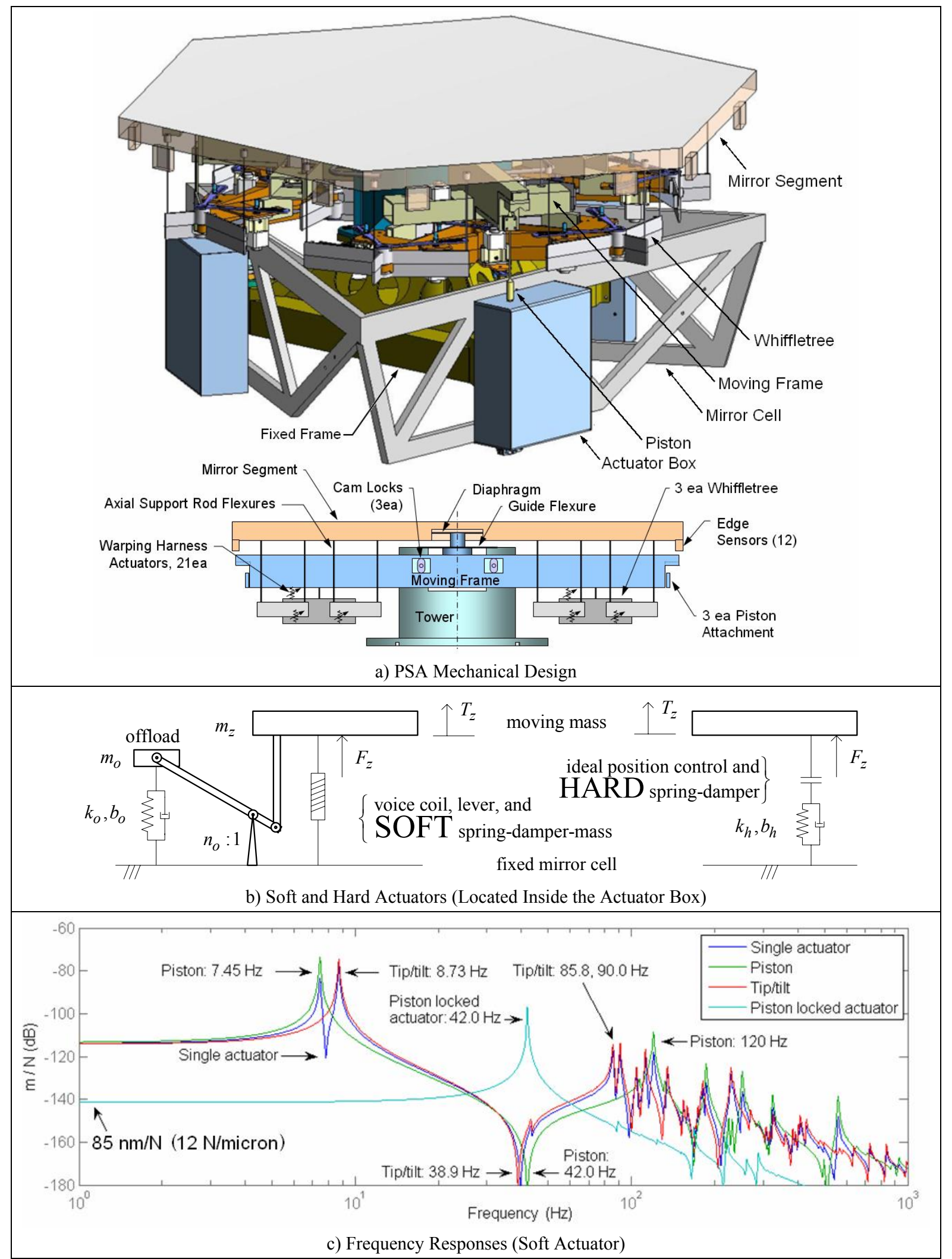

Figure 1: PSA Mechanical Design, Actuators, and Frequency Response 
Section 2 presents the goals and requirements for the PSA servo control loop, and Section 3 reviews the dynamics of the PSA and mirror cell. Section 4 presents the control system architecture and CSIE analysis methods. Section 5 contains instructional control designs using simplified models and literal analysis, used to set PSA bandwidth requirements and to help understand CSIE. Section 5 is a trade study of two PSA compensators using finite element models, one optimized for rigid base performance, and the second modified to reduce CSIE.

\section{GOALS AND REQUIREMENTS}

The M1CS control system architecture has a set of PSA inner loops using actuator position sensors and a M1CS outer loop using edge sensors. The PSA inner loop controller is defined by a set of performance and stability requirements.

Performance: The relative stiffness of a PSA mounted on the mirror cell to a force (wind) acting on the segment shall be greater than $10 \mathrm{~N} /$ micron for frequencies $1 \mathrm{~Hz}$ and below. The response above $1 \mathrm{~Hz}$ shall be minimized. The relative stiffness is defined using the motion of the forced segment relative to its neighbors.

Stability: The PSA control loop shall be stable when operating individually on a rigid base and when used in combination mounted on the mirror cell. Robustness margins measured at the force input shall be no less than plus or minus $10 \mathrm{~dB}$ and 35 degrees phase margin. These margins are to be maintained respectively at every -180 degree crossover and every unit magnitude crossover, thereby taking into account potential stability problems due to low frequency mirror cell modes. Structural resonance peaks in the loop transfer function above the PSA bandwidth shall be less than $-6 \mathrm{~dB}$.

Goals and requirements for the M1CS outer loop are presented in the companion paper [Ref. 2].

\section{CONTROLLED ELEMENT DESCRIPTION}

The controlled elements are the primary support assemblies (PSAs) and the mirror cell to which they are connected. Each is described and frequency responses presented. ${ }^{2}$

\subsection{Primary Segment Assembly}

The mechanical design for the PSA is shown in Figure 1a. The mirror segment is supported axially by a 27 point whiffletree and laterally via a central diaphragm. A moving frame isolates the central lateral diaphragm from the forces resulting from the required $5 \mathrm{~mm}$ of travel in piston. The three actuators control piston, tip, and tilt of the PSA and are located between the fixed frame and the moving frame.

Idealized models of the actuators are shown in Figure 1b. Two versions are shown: soft and hard. The soft actuator is a voice coil with an active spring-mass-damper offload system used to minimize power consumption by supporting the weight of the PSA. While the spring, damper, and lever are included in this analysis the offload mass is assumed zero. The hard actuator is a position controller with a "hard" spring, much stiffer than the PSA structure. The ideal version instantly obtains the commanded position.

Frequency responses of a finite element model of the PSA including a soft actuator are shown in Figure 1c. A damping ratio of $0.5 \%$ is assumed for all of the resonant modes. Piston and tip/tilt responses are from actuator force to moving mass position. In the piston response the three actuators move the same amount. In tip/tilt motion the center of the mirror segment does not move. Due to symmetry the frequency response is the same for any angle of tip/tilt. The single actuator response is a combination of piston and tip/tilt; which is why resonant modes from both piston and tip/tilt appear in the single actuator response. Resonances below $10 \mathrm{~Hz}$ are due to the offload spring.

The locked actuator response is also shown in Figure 1c, which is the response from force on the mirror segment to position of the mirror segment with the actuators locked in place. Just the piston response is shown. The dominant mode is at $42 \mathrm{~Hz}$, the same frequency as the zero in the actuator response. The static compliance of the PSA structure is 85 $\mathrm{nm} / \mathrm{N}$ (nano-meter/Newton). Compare this to three steel bolts $1 / 2$ inch in diameter and one foot long (modulus of elasticity $E=2.76 \mathrm{e} 7 \mathrm{lb} / \mathrm{sq}$-in) with compliance $L /(A \times E)=4.3 \mathrm{~nm} / \mathrm{N}$. The steel bolts are about 20 times as stiff.

A simple model of the locked actuator piston response is a lumped mass of $m=169 \mathrm{~kg}$ on one spring with a stiffness of $k=12 \mathrm{~N} /$ micron. The pole is at $f=\operatorname{sqrt}(k / m) /(2 \pi)=42 \mathrm{~Hz}$. The movable mass of the PSA including the mirror segment

\footnotetext{
${ }^{2}$ The PSA finite element model used in this paper is from Aug 2007. The TMT finite element model is version 70420, with the mass of the segments removed.
} 
is $203 \mathrm{~kg}$. A two-lumped mass model of the PSA piston motion uses the same spring between masses of $169 \mathrm{and} 33 \mathrm{~kg}$. The frequency response of the lumped mass model compares favorably with that of the finite element model. Figure 1c also illustrates that the majority of the dynamics above $50 \mathrm{~Hz}$ are associated with interactions between the moving frame and the whiffle-tree/segment assemblies.

\subsection{Mirror Cell}

The PSA actuator force reacts through the PSA fixed frame and through the mirror cell, which in turn reacts against the lower mirror cell support structure; eventually the load path is taken into the elevation rockers through the Az structure and into the pier and soil. A finite element model of the telescope, including the pier and soil, but not the PSAs on top of the mirror cell, is used to determine the static deflections and frequency responses from vertical force downward on the mirror cell to the deflected position of the mirror cell. Results are summarized in Figure 2. The telescope structure is shown in Figure 2a. The mirror cell is a relatively light truss structure (black in the Figure 2a) supported by a heavier truss like support structure (blue in the figure).

Static Deflections: Static deflections of the mirror cell are shown in Figure 2b. The top surface in this figure shows the result of pushing downward one-at-a-time with one Newton of force at virtual mirror cell nodes defined at the center of the PSA locations. The bottom surface shows the result of pushing with one Newton simultaneously on all of these nodes for a total of 492 Newtons of applied force.

The top line in Figure 2c is the same one-at-a-time deflections as in Figure 2b, but plotted versus the radial distance of the mirror cell nodes. The one-at-a-time deflections range from 30 to $81 \mathrm{~nm}$ with an average deflection of $43 \mathrm{~nm}$. The bottom line is the largest difference between neighbor segments, plotted versus the radial distance of the node that is pushed. These values range from 22 to $49 \mathrm{~nm}$ with an average of $27 \mathrm{~nm}$. Both of these lines increase at the outer radius of the M1 mirror where there is less structural support. The maximum difference between neighboring segments is a measure of high spatial frequency deflections. The $25 \mathrm{~nm}$ reference line is a goal of the structural design.

The shape of the M1 deflection in Figure 2b is different when the forces are applied simultaneously. The largest deflections are in the middle, rather than on the edge. The mirror cell deflections range from 765 to $1302 \mathrm{~nm}$ with an average deflection of $1104 \mathrm{~nm}(2.3 \mathrm{~nm} / \mathrm{N})$. The shape is primarily due to deflections of the mirror cell support structure. Consider the deflection in Figure $2 \mathrm{~b}$ along the $\mathrm{x}$-axis. The response is consistent with a uniform vertical force applied to a flexible beam supported at both ends.

Place the PSA's on top of the mirror cell and apply a downward force of one Newton on a single segment. The surface of the mirror segment deflects $85 \mathrm{~nm}$ relative to the mirror cell, and the PSA actuator deflection settles to zero if the control law contains an integrator. The surface of the underlying mirror cell deflects on average $43 \mathrm{~nm}$, and the largest difference between neighboring segment locations averages $27 \mathrm{~nm}$. The underlying mirror cell support structure deflects only about $2 \mathrm{~nm}$. An analogy is a waterbed: a push at one spot will create a dimple at the top of the mattress and a much smaller compression of the underlying frame.

Frequency Responses: Now consider the mirror cell frequency responses without the PSAs on top. In Figure $2 \mathrm{~d}$ the vertical force is applied at the center of the segment locations one-at-a-time. Eighty-two frequency responses are superimposed, covering a one-sixth pie shaped segment of the mirror cell. There are obvious differences in the 82 responses, but common to all save one is the prominent peak at about $54 \mathrm{~Hz}$. A one-mass model of this response has $1 / k$ $=40 \mathrm{~nm} / \mathrm{N}, f=54 \mathrm{~Hz}$, and mass $m=k /(2 \pi f)^{2}=217 \mathrm{~kg}$.

The piston (simultaneous force) response is shown in Figure 2e. A peak occurs at $5.4 \mathrm{~Hz}$. This is primarily the response of the mirror cell support structure and the underlying azimuth structure. A one-mass model of the piston response has $1 / k=2.3 \mathrm{~nm} / \mathrm{N}, f=5.4 \mathrm{~Hz}$, and mass $m=k /(2 \pi f)^{2}=3.78 \mathrm{e} 5 \mathrm{~kg}$. This one-mass model will be used to help understand control structure interaction.

The remaining responses are for Zernike basis vectors of radial degree 2,3, and 4. Given the mirror cell model $G_{c}$ with $n$ collocated inputs and outputs, and given a Zernike basis vector $b$, the Zernike response is $g_{c}=b^{T} G_{c} b / n$. Zernike basis vectors are used because they are the standard basis for analyzing the optical response of the telescope. As the Zernike radial degree increases, so does the frequency of the dominant resonance, indicating an approximate correspondence between the Zernike basis vectors and structural eigenvectors, and also an approximate decoupling of the Zernike responses. An assumption of decoupled Zernike responses is used to analyze control structure interaction. 


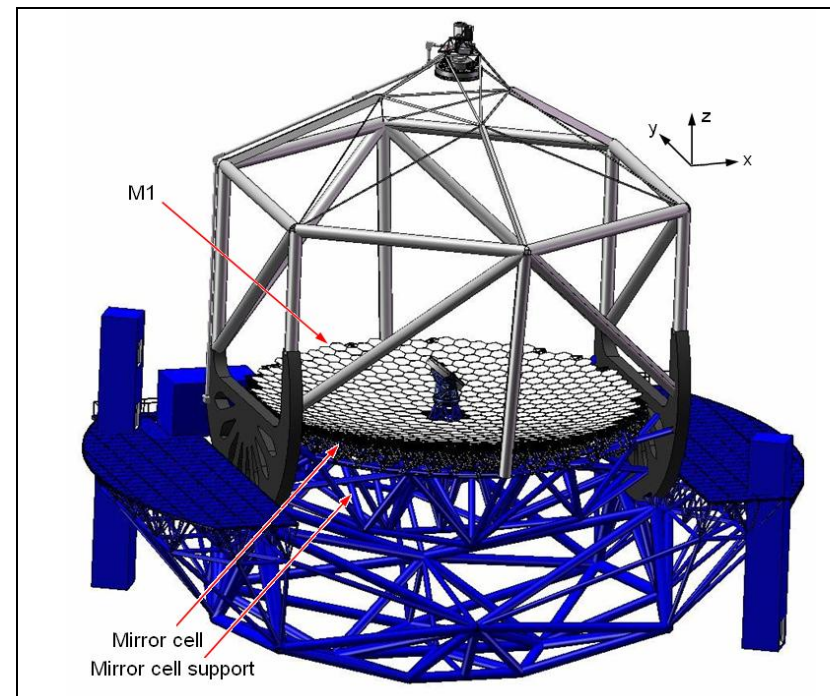

a) TMT Structure

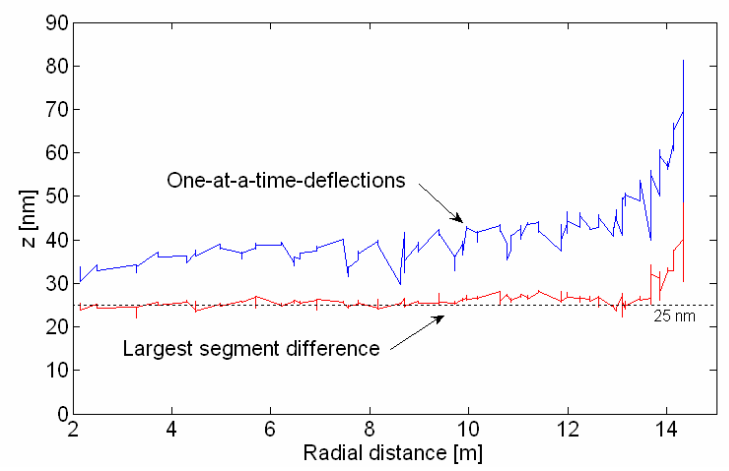

c) M1 Segment Static Deflections

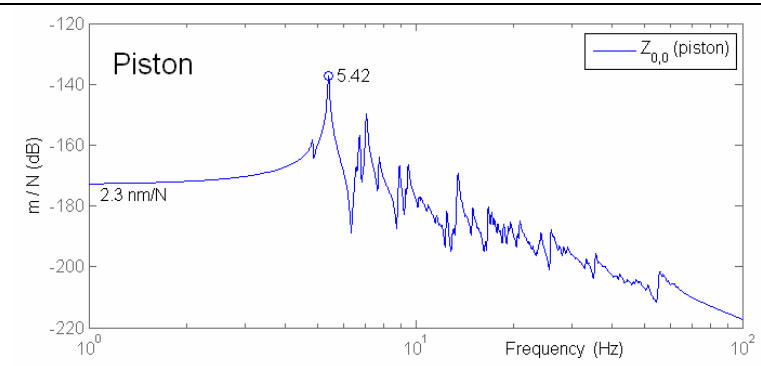

e) M1 Piston Frequency Response

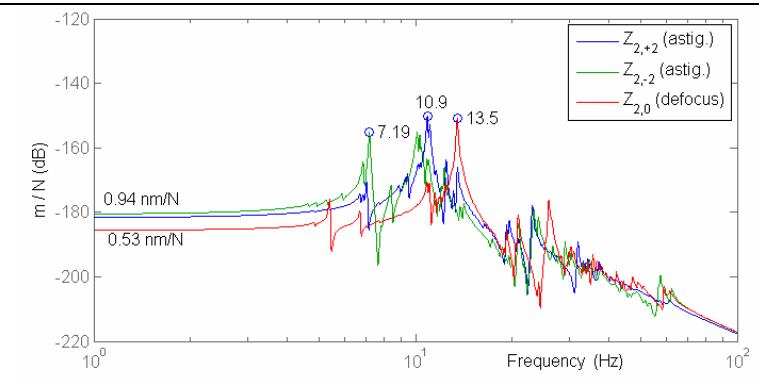

g) M1 Zernike Radial Degree 2

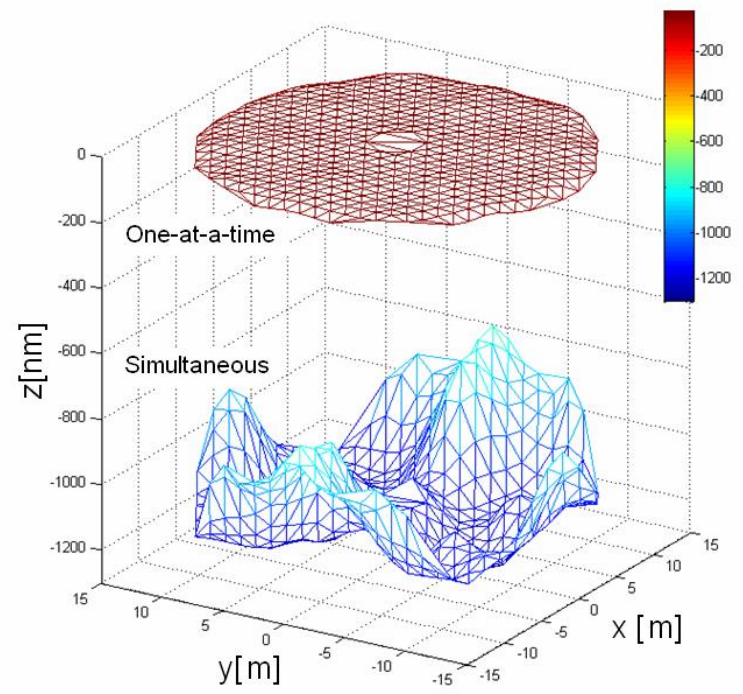

b) M1 Static Deflections

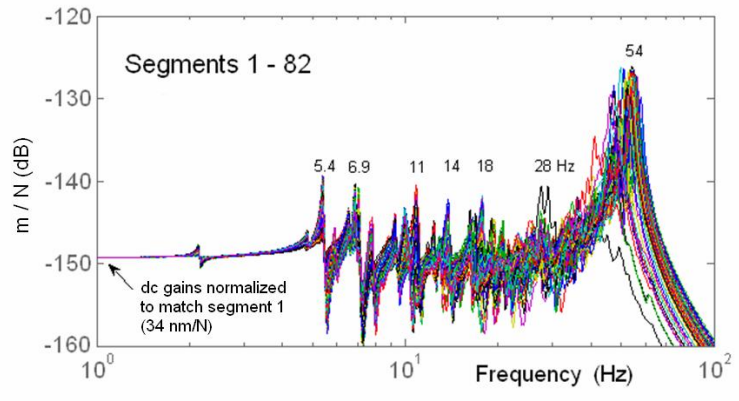

d) M1 Segment Frequency Responses

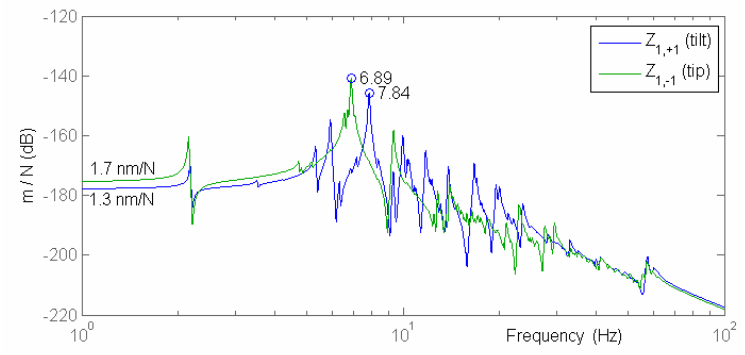

f) M1 Zernike Radial Degree 1

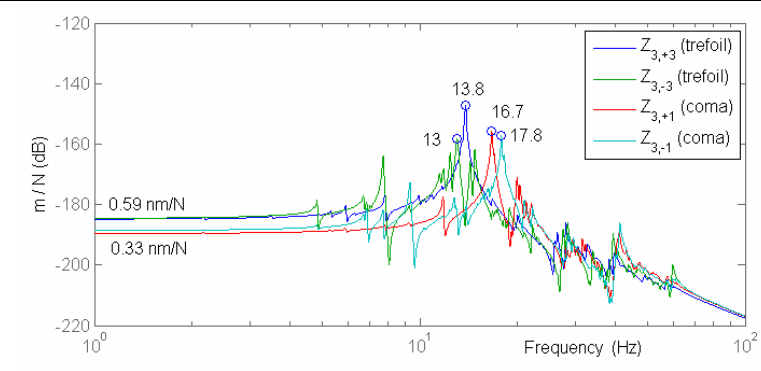

h) M1 Zernike Radial Degree 3

Figure 2: Mirror Cell Static Deflections and Frequency Responses 


\section{CONTROL ARCHITECTURE}

\subsection{Description}

The M1 control system architecture is shown in Figure 3. The signals are defined in Table 1. Each has dimension $1476 \times 1$. Translations use letter $T$ and have units of meters. Forces use letter $F$ and have units of Newtons. The system and controller blocks are defined in the figure and each has dimension $1476 \times 1476$. The PSA structure $G(s)$ is block diagonal. The mirror cell model has the general form $G_{c}(s)=$ $\Phi^{\mathrm{T}}\left(s I+\Omega^{2}\right)^{-1} \Phi$, where $\Omega$ is diagonal and contains the structural modes. The mirror cell model includes the entire telescope except for the PSAs. The mirror cell model $G_{c}(s)=0$ for the case of the PSAs on a rigid base. There are several controller options that fit within the general structure shown in Figure 3: 1) all of the controller blocks can be diagonal, 2) the PSA inner loop can be block diagonal, allowing separate control of PSA piston and tip/tilt motion, and 3) either or both the PSA and M1CS loops can be defined using basis vectors for modal control, allowing different bandwidth for low and high spatial frequency modes. The outer loop using edge sensors is not closed in this paper and is treated in the companion paper [Ref. 2].
Table 1: Signals

\begin{tabular}{|l|l|}
\hline & Definition \\
\hline$T_{w}$ & Mirror segment positions \\
\hline$T_{z}$ & Moving mass positions \\
\hline$T_{c}$ & Mirror cell positions \\
\hline$T_{w}-T_{c}$ & Relative mirror segment pos. \\
\hline$T_{z c}=T_{z}-T_{c}$ & Relative moving mass pos. \\
\hline$T_{z c m d}$ & PSA commanded position \\
\hline$T_{m c}$ & M1 commanded position \\
\hline$F_{w}$ & Force on mirror segments \\
\hline$F_{z}$ & Actuator force \\
\hline
\end{tabular}

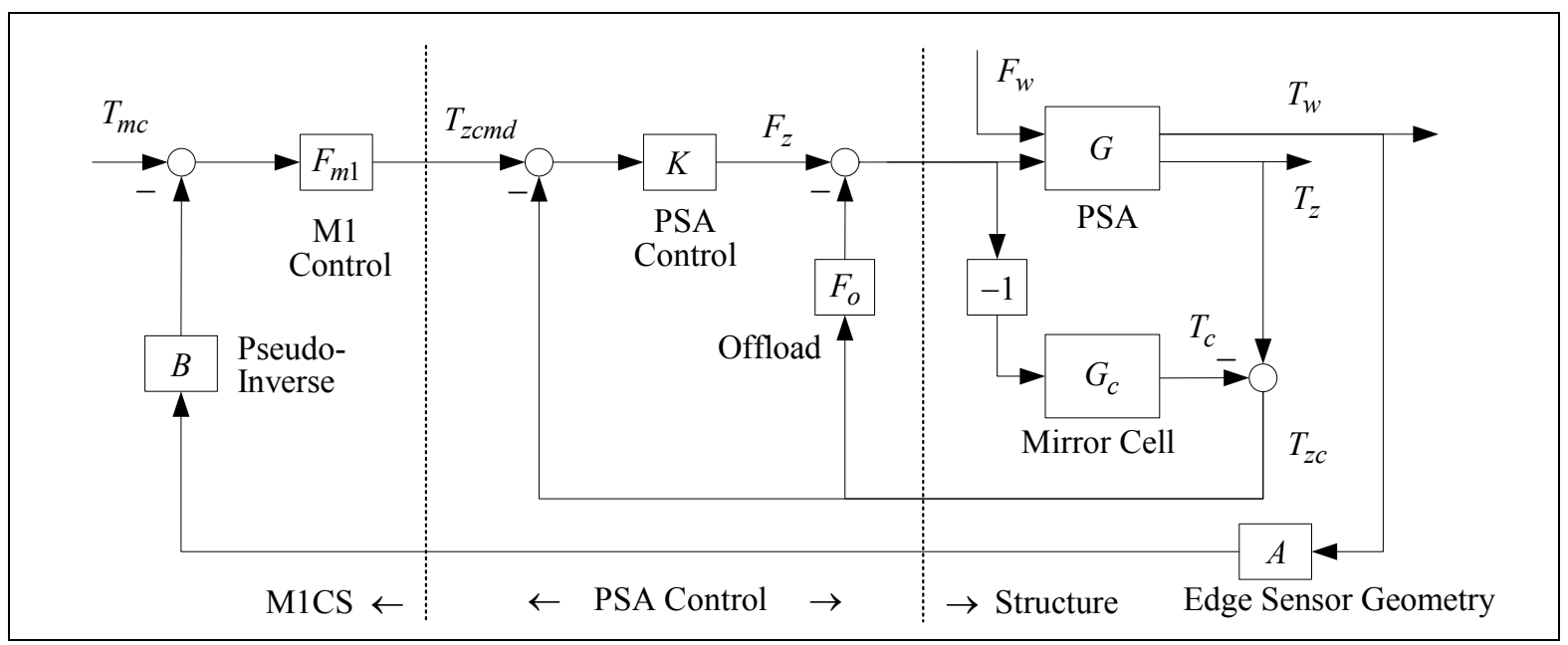

Figure 3: M1 Control System

\subsection{Analysis Method for Control Structure Interaction}

The closed loop control system will change the mirror cell modes, potentially reducing the damping or destabilizing one or more of these modes. It is important to understand why this happens and then design the control system to prevent this occurrence. The approach used in this paper is to approximate the MIMO system with a set of SISO systems. The resulting SISO systems provide insight into the CSIE and more importantly provide a computationally efficient means for designing the PSA compensator. Multivariable methods (using state space models and singular values) are used in the companion paper to verify stability and robustness.

Piston response (and scaling): A simple model of the mirror cell piston response is a rigid body connected by a spring to ground. The SISO transfer function model with force input and position output is $g_{c}=1 /\left(m_{c} s^{2}+b_{c} s+k_{c}\right)$. A MIMO version of the same rigid body is a set of points on the rigid body, where force applied at any point results in the same response at all of the points. The MIMO transfer function is $G_{c}=g_{c} u u^{T}$, where $u$ is a length $n$ vector of ones. It is more convenient to work with unit-norm vectors, in which case $G_{c}=n g_{c} b b^{T}$, where $b=u / \operatorname{sqrt}(n)$. Now work backwards from $G_{c}$ to recover the SISO model $g_{c}=b^{T} G_{c} b / n$. This way of defining a SISO response is generalized for any unitnorm vector $b$, and it is noted that in order to be consistent with the MIMO piston response the scale factor $n$ is included. 
Zernike responses (and decoupling): The MIMO model of the mirror cell has the form $G_{c}(s)=\Phi^{\mathrm{T}}\left(s \mathrm{I}+\Omega^{2}\right)^{-1} \Phi$, with $N$ modes and $n$ inputs and outputs. Define an $n \times n$ matrix $B$ where each column $b_{i}$ is a unit-norm Zernike vector, and defined the following Zernike responses:

$$
\begin{aligned}
& g_{c i}=b_{i}^{T} G_{c} b_{i} / n=\text { SISO Zernike response } \\
& g_{c i j}=b_{i}^{T} G_{c} b_{j} / n=0 \text { (assume decoupled) }
\end{aligned}
$$

The decoupling is approximately true for the mirror cell. The matrix $B$ of Zernike vectors is unitary, meaning $B B^{T}=I$, hence $G_{c}=B B^{T} G_{c} B B^{T}$, and with the assumption of decoupling it follows:

$$
G_{c}=\sum_{i=1}^{n} n g_{c i} b_{i} b_{i}^{T}
$$

In this way the MIMO response $G_{c}$ is replaced by a sum of SISO Zernike responses $g_{c i}$, each scaled by $n$ and multiplied by the $n \times n$ block $b_{i} b_{i}{ }^{T}$.

Closed loop response (using one Zernike): Select one Zernike response and replace the mirror cell model with just the response: $G_{c} \approx n g_{c} b b^{T}$. A block diagram of the closed loop system is shown in Figure 4 .

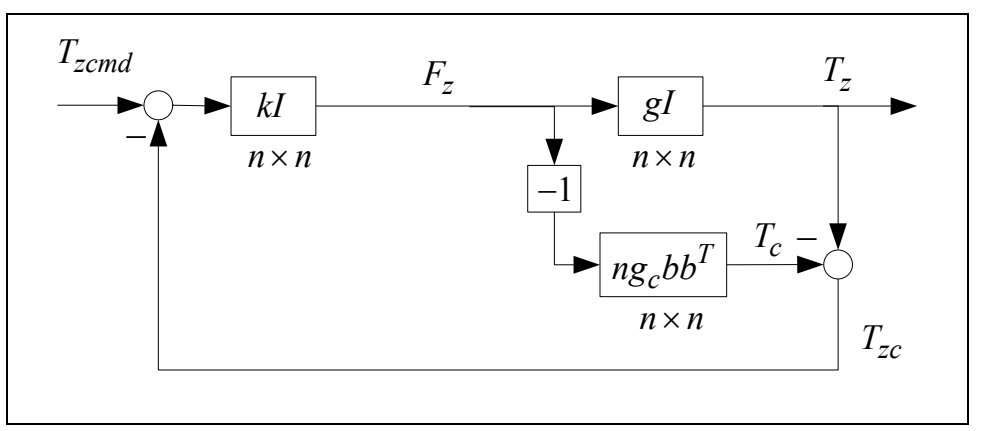

Figure 4: PSA Inner Loop with Mirror Cell Approximated using One Zernike (and later with a sum of Zernikes)

The closed loop command response is:

$$
T_{z}=g k[\underbrace{(1+g k)}_{\alpha} I+\underbrace{n g_{c} k}_{\beta} b b^{T}]^{-1} T_{z c m d}
$$

The $n \times n$ matrix in the square brackets in (3) needs to be inverted. The matrix has a special form that makes it possible to use the matrix inversion lemma (MIL) ${ }^{3}$ :

$$
\left(\alpha I+\beta b b^{T}\right)^{-1}=\alpha^{-1}-\beta b b^{T} \alpha^{-1}(\alpha+\beta)^{-1}
$$

The $n \times n$ matrix inverse is replaced with two $1 \times 1$ inverses. The computational saving is significant: calculations that take about 30 minutes are reduced to less than one second. Furthermore, the poles of the closed loop system are the zeros of the return difference equations (RDEs) $\alpha=1+g k$ and $\alpha+\beta=1+g k+n g_{c} k$. The controller $k$ must stabilize the poles for the rigid base, these are the poles defined by $\alpha$, and must also stabilize the new set of poles defined by $\alpha+\beta$. Thus it is seen that mounting the PSA on a flexible mirror cell results in an additional condition that must be satisfied by the PSA controller.

An input/output response can be defined that just has the "new" poles, where $T_{z}=b T_{z b}, T_{z c m d b}=b^{T} T_{z c m d}$, and:

$$
\frac{T_{z b}}{T_{z c m d b}}=g k b^{T}\left(\alpha I+\beta b b^{T}\right)^{-1} b=\frac{g k}{1+g k+n g_{c} k}
$$

The piston response defined as in equation (5) is used in Section 5.3 to demonstrate the CSIE stability problem.

${ }^{3}$ The MIL is $(A+U C V)^{-1}=A^{-1}-A^{-1} U\left(C^{-1}+V A^{-1} U\right)^{-1} V A^{-1}$. Set $A=\alpha I, U=\beta b, C=I$, and $V=b^{T}$. 
Closed loop response (using all Zernikes): The closed loop response using the diagonal form (2) of $G_{c}$ is:

$$
T_{z}=g k[\underbrace{(1+g k)}_{\alpha} I+\sum_{i=1}^{n} \underbrace{n g_{c i} k}_{\beta_{i}} b_{i} b_{i}^{T}]^{-1} T_{z c m d}
$$

The MIL is applied $n$ times to obtain:

$$
\left(\alpha I+\sum_{i=1}^{n} \beta_{i} b_{i} b_{i}^{T}\right)^{-1}=\alpha^{-1}-\sum_{i=1}^{n} \beta_{i} b_{i} b_{i}^{T} \alpha^{-1}\left(\alpha+\beta_{i}\right)^{-1}
$$

The SISO Zernike responses nicely separate, and the controller $k$ must stabilize the closed loop poles for all of these responses. There are a large number of SISO responses to be checked, and in practice just a subset of the Zernike responses is used. A controller $k$ that stabilizes a subset of Zernike responses, and even the full set, does not guarantee that the actual closed loop system is stable. The restriction occurs because the assumed diagonal form (2) of $G_{c}$ is only approximate, and this is why additional analysis methods are needed to formally verify stability and robustness.

\section{INSTRUCTIONAL CONTROL DESIGNS}

Three control designs are presented to develop an understanding of the PSA and mirror cell dynamics.

\subsection{Design 1: Required PSA Bandwidth}

The first design is an analytical study used to determine the required bandwidth of the PSA controller. The "bandwidth" $f_{c}$ is defined in this paper as the unit magnitude crossover of the loop transfer function (ltf). Model a single PSA on a rigid base as a lumped mass of $m_{t}=203 \mathrm{~kg}$, with no offload spring, so that $T_{z} / F_{z}=g(s)=1 /\left(m_{t} s^{2}\right)$. The proportionalintegral-derivative (PID) compensator is $f_{\text {pid }}=k_{r}\left(\mathrm{~s}^{2}+k_{p} \mathrm{~s}+k_{i}\right) / \mathrm{s}$. The PID gains are computed based on the following performance and robustness parameters:

$$
\begin{array}{ll}
f_{c d}[\mathrm{~Hz}]=\text { desired unit magnitude crossover frequency } & \text { PM }[\mathrm{deg}]=\text { phase margin } \\
f_{\operatorname{lgm}}[\mathrm{rad} / \mathrm{sec}]=-180 \text { deg crossover where LGM occurs } & \text { LGM }[\mathrm{dB}]=\text { lower gain margin }
\end{array}
$$

The phase margin is extra phase lag that destabilizes the system, and the lower gain margin is the gain reduction that destabilizes the system. The PID gains based on these parameters are:

$$
\begin{aligned}
k_{i} & =\omega_{\lg m}^{2}=-\beta \omega_{c d}^{2} /\left(\operatorname{lgm} \times \operatorname{sqrt}\left(1+\beta^{2}\right)\right) & & \mathrm{pm}=\mathrm{PM} \times \pi / 180, \operatorname{lgm}=10^{\mathrm{LGM} / 20} \\
k_{p} & =\beta\left(k_{i}-\omega_{c d}^{2}\right) / \omega_{c d} & \text { where } & \beta=\tan (\mathrm{pm}+\pi / 2) \\
k_{r} & =\operatorname{lgm} \times m_{t} \times k_{i} / k_{p} & & \omega_{c d}=2 \pi f_{c d}, \omega_{\operatorname{lgm}}=2 \pi f_{\operatorname{lgm}}
\end{aligned}
$$

Either $f_{\text {lgm }}$ or LGM can be used to set the integral gain. Note that the rate gain varies with the mass of the PSA. The parameter values $\mathrm{PM}=45 \mathrm{deg}$ and $\mathrm{LGM}=12 \mathrm{~dB}$ result in high gain below $f_{c d}$ but with allowance for phase lag due to a structural filter. The mirror segment stiffness requirement is:

$$
\left|T_{z} / F_{z}\left(j \omega_{d}\right)\right| \approx \omega_{d} /\left(k_{r} k_{i}\right)=10^{-7} \text { for } \omega_{d}=2 \pi[\mathrm{rad} / \mathrm{sec}]
$$

Substitute (9) into (10) and solve for $f_{c d}$ :

$$
f_{c d}=\frac{1}{2 \pi}\left[\frac{10^{7} \omega_{d}(\operatorname{lgm}-\cos (\mathrm{pm}))}{m_{t} \cos (\mathrm{pm}) \sin (\mathrm{pm})}\right]^{1 / 3} \approx 20 \mathrm{~Hz}
$$

Use $f_{c d}=20 \mathrm{~Hz}$ for the desired unit magnitude crossover frequency. The actual bandwidth $f_{c}$ will exactly equal $f_{c d}$ for the inertial system $g(s)=1 /\left(m_{t} s^{2}\right)$, but as the system model becomes more complicated by adding structural resonances then $f_{c} \approx f_{c d}$, and adjustments are made to $f_{c d}$ until the desired $f_{c}$ is achieved. The control system architecture and resulting frequency responses are similar to those in Design 2, without the resonance, and not separately plotted. 


\subsection{Design 2: Single PSA on a Rigid Base (Comparison Hard versus Soft Actuators)}

Hard and soft actuators are compared using the piston response of a single PSA mounted on a rigid base, using the twomass model shown in Figure 5a. The figure includes the parameters and the feedback architecture.

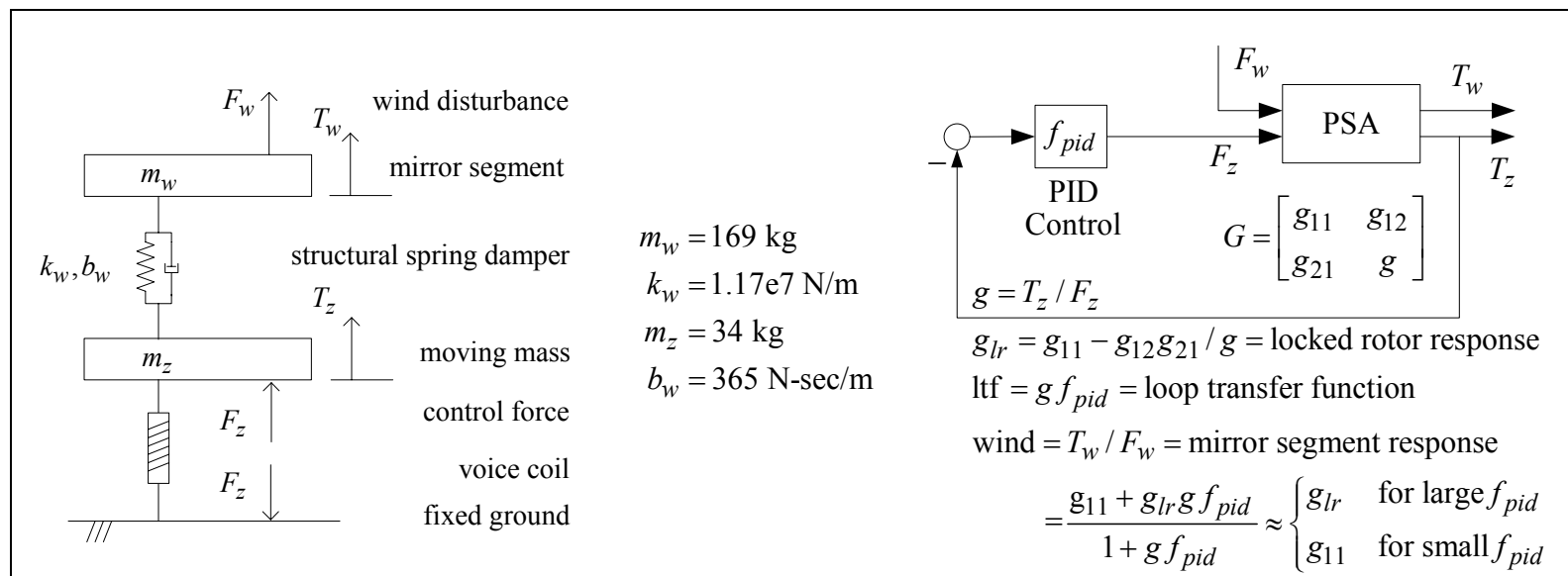

a) Model, parameters, and feedback architecture

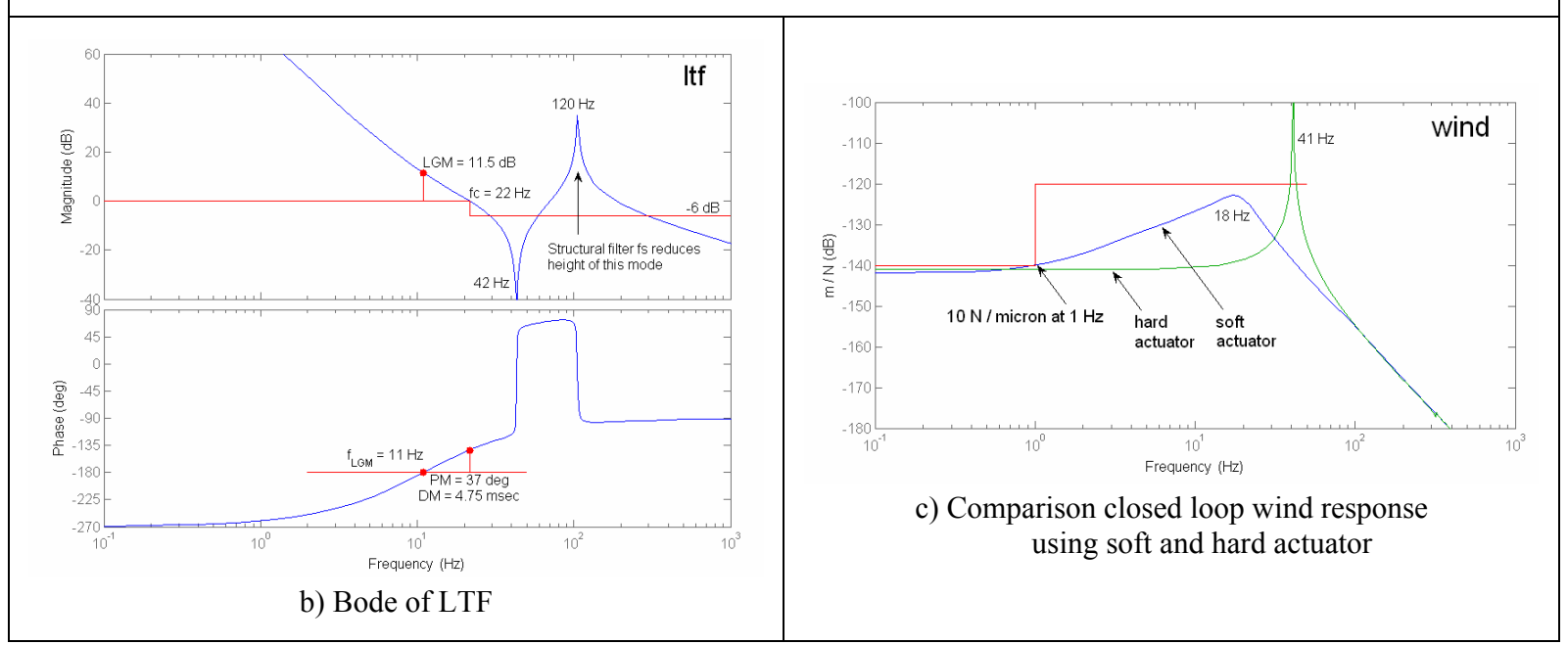

Figure 5: Design 2 Survey: Hard Versus Soft

Soft actuator: For the soft actuator design just the voice coil is used. The offload spring-damper adds to the PID compensator and for simplicity is not included in the instructional designs. The control architecture is shown in Figure $5 \mathrm{~b}$, which includes definitions of the ltf and wind responses, along with approximations of the wind response. For the PID design use $\mathrm{PM}=45 \mathrm{deg}, \mathrm{LGM}=12$, and $f_{c d}=25 \mathrm{~Hz}$. The ltf is shown in Figure $5 \mathrm{~b}$. The achieved bandwidth is $f_{c}=$ $22 \mathrm{~Hz}$ and phase margin is $\mathrm{PM}=37 \mathrm{deg}$. The differences are due to the nearby locked actuator zero at $42 \mathrm{~Hz}$. The closed loop wind response is shown in Figure $5 \mathrm{c}$ and it is seen that the response falls below the "corner" of $-140 \mathrm{~dB}$ and $1 \mathrm{~Hz}$.

The wind response using the soft actuator is above $-140 \mathrm{~dB}$ from $1 \mathrm{~Hz}$ to $40 \mathrm{~Hz}$, peaking at $-123 \mathrm{~dB}$ at $18 \mathrm{~Hz}$, a gain increase of 7:1. This increase is the "cost" of using a soft actuator. The von Karman wind model [Ref. 3] has a corner frequency at roughly $0.2 \mathrm{~Hz}$ (wind speed of $1 \mathrm{~m} / \mathrm{s}$ and turbulence outer scale of $5 \mathrm{~m}$ ) with half the energy below $0.2 \mathrm{~Hz}$, with little wind energy above $1 \mathrm{~Hz}$. The expected acoustic environment in the enclosure is under investigation to determine if the response above $1 \mathrm{~Hz}$ needs adjustment.

Hard actuator: The force applied to the moving mass $m_{z}$ is due to compliance of the hard actuator modeled as a spring with compliance $k_{h}$. To analyze the wind response of the hard actuator use $f_{p i d}=k_{h}=10 \times k_{w}$. The resulting wind response is shown in Figure $5 \mathrm{c}$, which has a prominent peak at $f_{h d}=41 \mathrm{~Hz}$. The wind response is essentially the same as the 
locked actuator response shown in Figure 1c. Increased values of $k_{h}$ will bring $f_{h d}$ closer to $f_{l a}=42 \mathrm{~Hz}$, but not above $f_{l a}$, and so there is little value in further increasing the stiffness of the hard actuator.

The hard actuator is better below $40 \mathrm{~Hz}$, but the peak at $41 \mathrm{~Hz}$ is a potential problem. Narrowband vibrations $10 \%$ or more away from $41 \mathrm{~Hz}$ will not excite this resonance. Disturbance with impulsive and sharp edged time responses have a broadband frequency response, and it is these types of disturbances that can cause ringing of the PSA at $41 \mathrm{~Hz}$.

\subsection{Design 3: Control Structure Interaction (Problem and Solution)}

In the final instructional control example the CSIE is demonstrated using the piston response of the mirror cell, modeled as shown in Figure 6a. A surprisingly low order approximation of the PSA and mirror cell dynamics suffices to demonstrate the problem. Fuse all of the mirror segments together to form a single large segment, and replace the mirror cell with a spring-mass-damper. Define:

$$
(1 / n) g(s)=1 /\left(n m_{t} s^{2}\right), g_{c}(s)=1 /\left(m_{c} s^{2}+b_{c} s+k_{c}\right)
$$

The controlled element changes to:

$$
(1 / n) g(s)+g_{c}(s)=\frac{\left(n m_{t}+m_{c}\right) s^{2}+b_{c} s+k_{c}}{\left(m_{t} s^{2}\right)\left(m_{c} s^{2}+b_{c} s+k_{c}\right)}
$$

Define the mass ratio $\alpha=n m_{t} / m_{c}$. The combined system differs from the rigid base version in two ways: 1) a complex dipole appears (a complex pole / zero pair) with the pole at $f_{c}=5.4 \mathrm{~Hz}$ and the zero at the lower frequency $f_{c} / \operatorname{sqrt}(1+\alpha)$, and 2) the high frequency gain increases by $(1+\alpha)$. Either of these effects can be a stability problem. The complex dipole working in conjunction with the integrator in the compensator can destabilize the mirror cell piston mode and is considered to be the more serious problem.

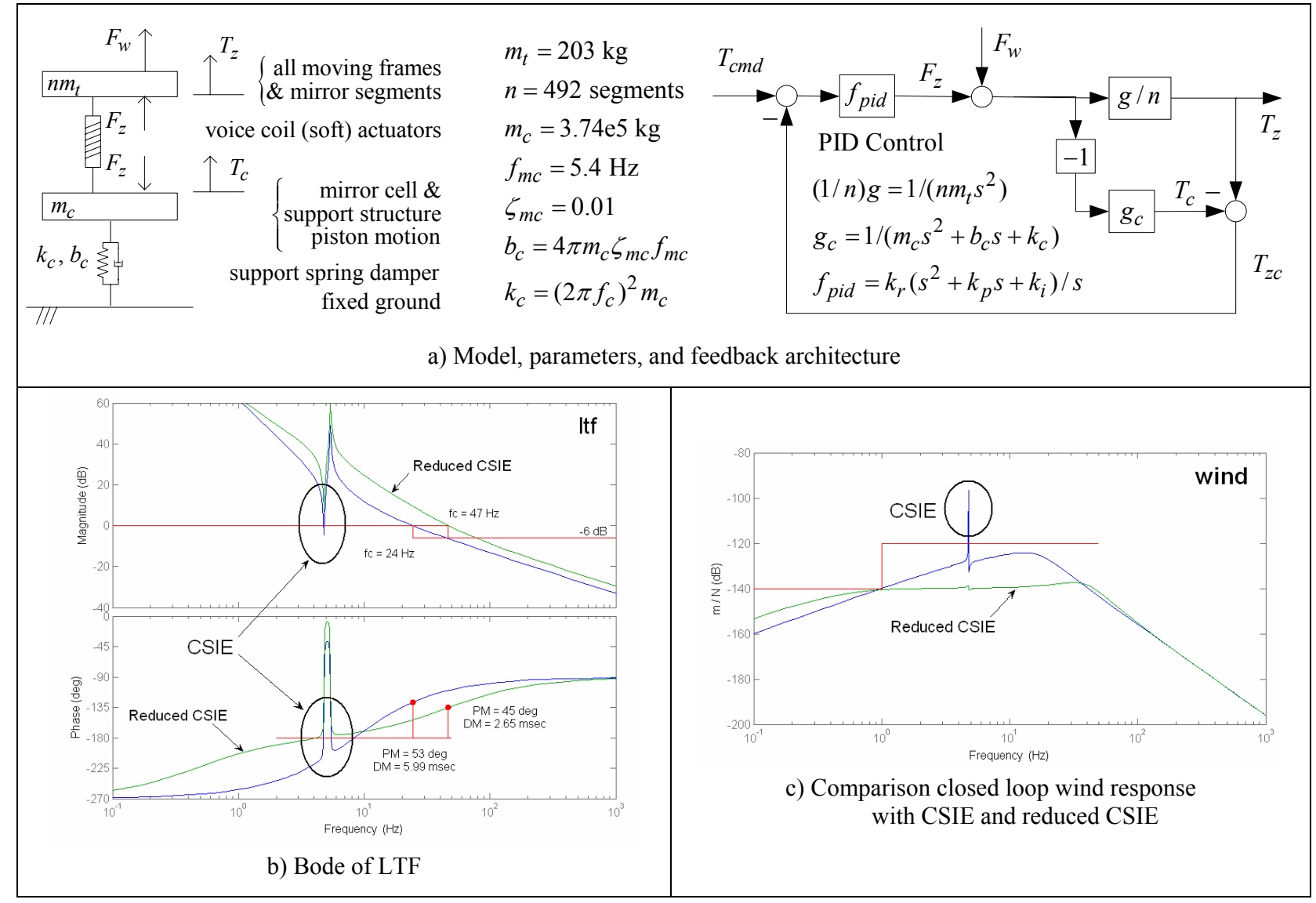

Figure 6: Design 3 Survey: CSIE Problem and Solution 
Problem: A PID controller is designed using the parameters $\mathrm{PM}=45 \mathrm{deg}, \mathrm{LGM}=12 \mathrm{deg}$, and $f_{c d}=20 \mathrm{~Hz}$. The results are surveyed in Figure $6 \mathrm{~b}$ and c. The achieved crossover increases to $24 \mathrm{~Hz}$ due to the extra gain above $5.4 \mathrm{~Hz}$ caused by the mass ratio $\alpha=0.26$. The wind response corner at $1 \mathrm{~Hz}$ is achieved. (The wind response decreases below $1 \mathrm{~Hz}$ because the controlled element is rigid). CSIE is seen in both the ltf and closed loop wind responses. Every -180 degree crossover and every unit magnitude crossover in the ltf Bode plot is a potential stability problem and it is seen in Figure $6 \mathrm{~b}$ there are several such crossovers near the $5.4 \mathrm{~Hz}$ mirror cell mode. The CSIE is visually more obvious in the closed loop wind response because lightly damped modes appear as spikes, and one such spike is clearly seen at $5.4 \mathrm{~Hz}$. An examination of the closed loop poles (not shown) indicates the closed loop system is stable, but the lightly damped mirror cell motion will probably cause image deterioration.

Solution: Stability of the mirror cell mode $f_{m c}$ can be guaranteed if the -180 degree crossover occurs at a frequency below the mirror cell mode. In this way $f_{m c}$ remains "phase stable," which means the phase response near $f_{m c}$ remains above -180 degrees, and hence feedback will further damp the mode. The -180 degree crossover frequency $f_{\text {lgm }}$ depends on the integral gain $k_{i}$. Lower the integral gain until the robustness margins at $f_{\text {lgm }}$ are acceptable. The safest approach is to eliminate the integral gain completely. The cost of lowering or eliminating the integral gain is that the low frequency gain is reduced, and hence the wind response corner may not be satisfied. The bandwidth can be increased to recover this gain using PID parameters $\mathrm{PM}=40 \mathrm{deg}, \mathrm{LGM}=35 \mathrm{~dB}$, and $f_{c}=40 \mathrm{~Hz}$. The CSIE no longer causes a stability problem, and the wind response looks very good across the entire frequency range. This solution unfortunately cannot be implemented because the needed bandwidth of $f_{c}=40 \mathrm{~Hz}$ is too close to the PSA located actuator zero at $42 \mathrm{~Hz}$.

\section{BENCHMARK CONTROL DESIGNS}

Two designs are presented for the PSA controller using high order finite element models. The first is a cautionary design optimized for a rigid base without taking into account CSIE. The second design makes changes to the controller that significantly reduces the CSIE.

\subsection{Design 4: Optimized for a Rigid Base:}

Refer again to the block diagram in Figure 3. The force input is $F_{z}=\left[\begin{array}{lll}F_{z 1} & F_{z 2} F_{z 3}\end{array}\right]^{T}$, with similar definitions for $F_{w}, T_{z}$, and $T_{w}$. Performance is increased by using separate piston and tip/tilt compensators. The $3 \times 3$ controller takes the form:

$$
K=F_{S} F_{\text {pid }}=V^{-1} \operatorname{diag}\left(k_{p} k_{t t} k_{t t}\right) V \text {, where } V=\left[\begin{array}{ccc}
1 / 3 & 0 & 0 \\
0 & 1 / \sqrt{6} & 0 \\
0 & 0 & 1 / \sqrt{18}
\end{array}\right]\left[\begin{array}{ccc}
1 & 1 & 1 \\
0 & -1 & 1 \\
2 & -1 & -1
\end{array}\right]=\begin{aligned}
& \text { piston } \\
& \text { tilt }
\end{aligned}
$$

where:

$$
\begin{aligned}
& \text { Piston: } k_{p}=f_{p i d} \times f_{s}=\frac{1.664 \mathrm{e}+004[0.5528,10.87]}{(0)} \times \frac{45.7(26.61)[0.1,110][0.05,120]}{(31.83)[0.1,44](54.27)[0.2,120]} \\
& \text { Tip/tilt: } k_{t t}=f_{p i d} \times f_{s}=\frac{1.171 \mathrm{e}+004[0.5429,10.61]}{(0)} \times \frac{56.42(23.81)[0.1,90][0.05,120]}{(31.83)[0.1,40](48.56)[0.2,120]} \\
& \text { the shorthand form is defined by } a(b)[\zeta, f]=a(s+b /(2 \pi))\left[s^{2}+4 \pi \zeta s+(2 \pi f)^{2}\right]
\end{aligned}
$$

The structural filters contain a notch with a staggered pole zero to producing a significant gain reduction, another notch at $120 \mathrm{~Hz}$, a lead-lag filter around crossover, and an extra one-pole roll-off.

Design 4 Survey: A system surveys for a single PSA on a rigid base is shown in Figure $7 \mathrm{a}$ and $\mathrm{b}$. The phase margin is at 30 degrees to maximize the low frequency gain, and it is seen that the closed loop wind corner at $1 \mathrm{~Hz}$ is satisfied.

Flexible Base Survey: Place all of the PSAs on the mirror cell and examine the first six Zernike responses. The survey is shown in Figure 7c and d. In the ltf Bode it is seen there are numerous crossovers introduced by the Zernike responses, hence there is a strong potential for lightly damped modes and perhaps instability. The lightly damped modes are clearly seen as spikes in the closed loop response. A Nichols chart, not shown, confirms instability. The Zernike responses with dominant poles closer to $f_{\text {lgm }}=12 \mathrm{~Hz}$ are more of a stability problem than the piston response, because it is near $f_{\text {lgm }}$ that "extra" -180 crossovers are more likely to occur. 
Wind Response: At low spatial frequencies the deflection of the mirror cell and support structure is significantly larger than the deflection of the PSA, including actuators. This results in a high amplitude wind response at low spatial frequencies regardless of the achieved actuator stiffness. For this reason Figure $7 \mathrm{~d}$ is plotted using the position of the mirror segment with respect to the local mirror cell position (called "relative wind"). Fortunately for both seeing-limited and adaptive-optics relevant performance metrics, it is the high spatial-frequency response of the primary mirror that is most important. At high spatial frequencies, the mirror cell is relatively stiff and the performance is driven by the actuator compliance.

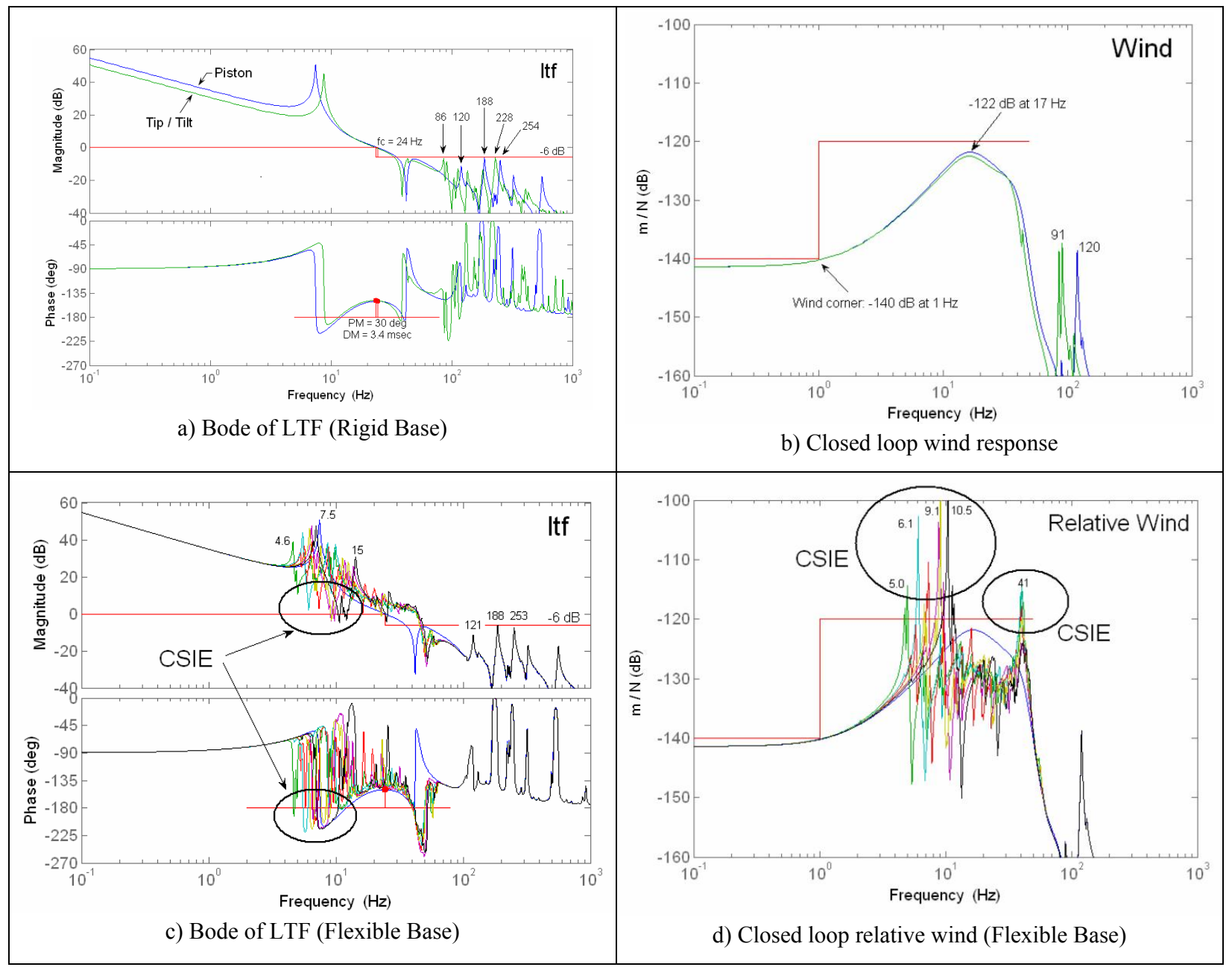

Figure 7: Design 4 Survey: Optimized for Rigid Base

Accelerometers and Extra Mass: Accelerometer measurements on the back side of the mirror segments have been studied. The advantage is stiffer closed loop wind response in the 1 to $40 \mathrm{~Hz}$ range, with a reduction in the peak response by about $6 \mathrm{~dB}$. The disadvantages are extra complexity, robustness with respect to sensor failures, and extra cost. An alternative is extra mass on the long side of the lever arm, which is equivalent to accelerometer feedback of the moving mass position, without the cost and complexity of an accelerometer.

\subsection{Design 5: Reduced CSIE}

An extra degree of freedom for the PSA control design is to include a damper on the long arm of the PSA actuator offload, large enough to significantly damp the PSA resonances. This damping can be achieved using a grounded voice coil. Use the following controller and addition damping for each PSA actuator, $K=\operatorname{diag}(k), F_{o}=b_{o} s$, where:

$$
k=\frac{888.9[0.9865,6.249](67.56)(6602)}{(0.1)(20.78)(1000)}, b_{o}=2667[\mathrm{~N}-\mathrm{sec} / \mathrm{rad}]
$$


The PID integrator is moved out to $0.1 \mathrm{~Hz}$, a lead-lag is included, and high frequency roll-off. The offload damping parameter is divided by 49 when placed on the long arm of the 7:1 lever. The loop transfer function and closed loop command response are surveyed in Figure 8 using the rigid base and the first six Zernikes. In the ltf plot the extra damping is included with the compensator.

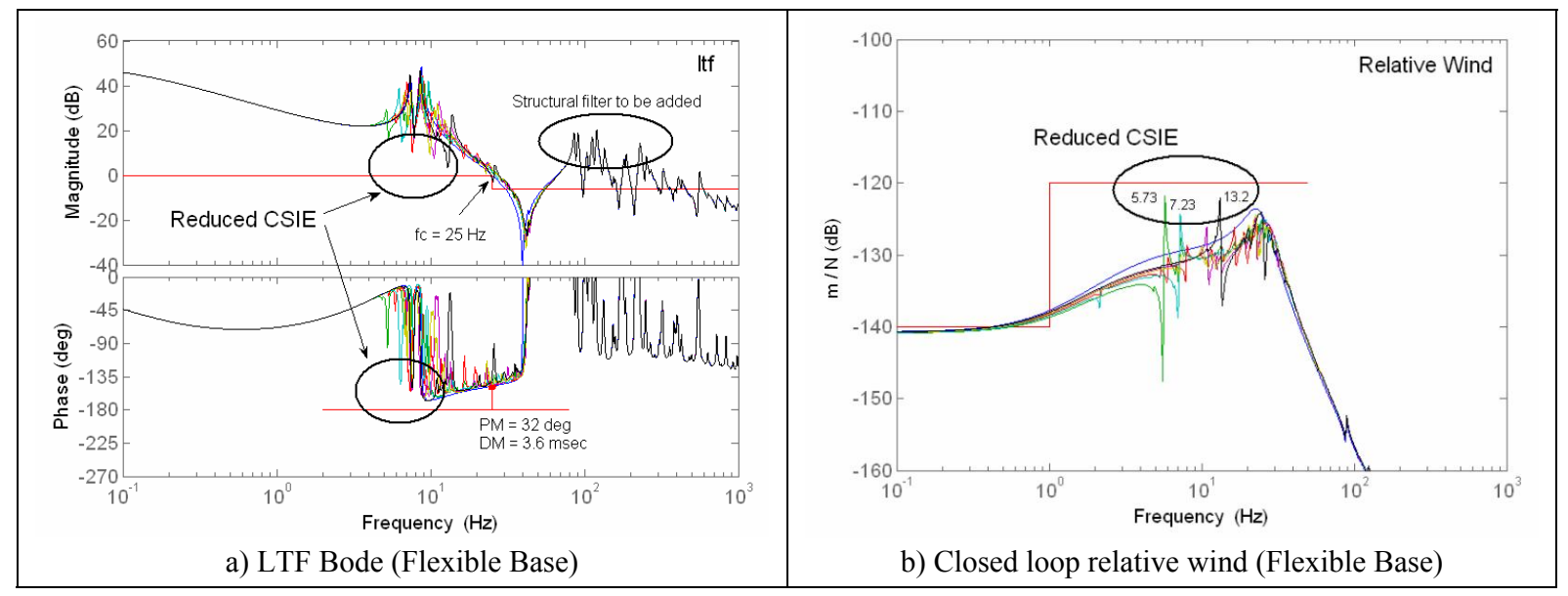

Figure 8: Design 4 Survey: Reduced CSIE

It is seen that the extra damping and moving the PID integrator has dramatically reduced the CSIE. Structural filtering is not included in this compensator and will be added in later design studies. The $1 \mathrm{~Hz}$ wind "corner" is missed by $2 \mathrm{~dB}$, and it is expected by 2 or 3 more $\mathrm{dB}$ when the structural filter is added. This is the PSA controller design that is carried forward in the companion paper [Ref. 2].

\section{CONCLUSIONS}

A set of control designs is presented that demonstrates an understanding of the control structure interaction effect and the ability to achieve performance requirements for the PSA inner loop control system. The last design is carried forward to the companion paper [Ref. 2] where multivariable methods are used to analyze stability, robustness, and M1 outer loop performance.

Finite element models are available for individual primary support assemblies and for the entire telescope. Static deflections and frequency responses using these models are presented and discussed. The frequency responses are the starting point for the PSA control system design. The main objective of the PSA controller is to meet a closed loop stiffness requirement.

The frequency responses of the FE models are combined in order to analyze control structure interaction. A method is developed for approximating the multivariable responses with a set of single input single output Zernike responses. A simple lumped mass model is used to show how CSI can destabilize a mirror cell mode and then how to change the PSA control system to reduce or eliminate adverse CSI. The change is to reduce the integral gain. A balance must be made between closed loop stiffness, bandwidth, and robustness.

An important ratio for CSIE analysis is $\alpha=n \times m_{t} / m_{c}$, the total moving mass of the PSA divided by the effective mass of the mirror cell. Each Zernike response has a different effective mass $m_{c}$. Ltf and closed loop wind responses are defined for each Zernike and used to determine if the resonant modes in the Zernike response are a potential stability problem.

Further study is underway for the PSA and M1 control systems. The issues being explored include 1) extra damping on the long side of the soft actuator level, 2) extra mass on the long side of the soft actuator level, 3) moving the voice coils to the long side, and 3) modal control for the PSA inner loop, 4) detailed modeling of both the soft and hard actuator dynamics. 


\section{ACKNOWLEDGEMENT}

The authors gratefully acknowledge the support of the TMT partner institutions. They are the Association of Canadian Universities for Research in Astronomy (ACURA), the California Institute of Technology and the University of California. This work was supported as well by the Gordon and Betty Moore Foundation, the Canada Foundation for Innovation, the Ontario Ministry of Research and Innovation, the National Research Council of Canada, the Natural Sciences and Engineering Research Council of Canada, the British Columbia Knowledge Development Fund, the Association of Universities for Research in Astronomy (AURA) and the U.S. National Science Foundation.

\section{REFERENCES}

[1] M. Troy, G. Chanan, S. Michaels, R. Bartos, G. Bothwell, R. Hein, M. Radin, J. Roberts, J. M. Rodgers, L. M. Scherr, B.-J. Seo, and D. Zimmerman, "A Conceptual Design for the Thirty Meter Telescope Alignment and Phasing System," SPIE Astronomical Telescopes and Instrumentation, Marseilles, France, 23-28 June 2008, SPIE 7012-125.

[2] MacMynowski, D. G., Thompson, P. M., and Sirota, M. J., “Analysis of TMT Primary Mirror Control-Structure Interaction”, SPIE Astronomical Telescopes and Instrumentation, Marseilles, France, 23-28 June 2008, SPIE 7017-41.

[3] MacMynowski, D. G., Blaurock, C., and Angeli, G. Z., "Dynamic Analysis of TMT”, SPIE Astronomical Telescopes and Instrumentation, Marseilles, France, 23-28 June 2008, SPIE 7017-31 